\title{
Interesting magnetic behavior from reduced titanium dioxide nanobelts
}

\author{
S. V. Chong, ${ }^{1, a)}$ K. Kadowaki, ${ }^{1}$ J. Xia, ${ }^{2}$ and H. Idriss ${ }^{3}$ \\ ${ }^{1}$ Institute of Materials Science, University of Tsukuba, 1-1-1 Tennodai, Tsukuba, Ibaraki 305-8573, Japan \\ ${ }^{2}$ Industrial Research Limited, P.O. Box 31310, Lower Hutt 5040, New Zealand \\ ${ }^{3}$ Department of Chemistry, The University of Auckland, Private Bag 92019, Auckland 1142, New Zealand
}

(Received 8 May 2008; accepted 21 May 2008; published online 11 June 2008)

\begin{abstract}
The magnetic behavior of titanium dioxide nanobelts has been investigated with and without Co doping. Room temperature ferromagnetism was observed when the Co-doped anatase $\mathrm{TiO}_{2}$ nanobelts were prepared via vacuum annealing of 2.5 at. \% Co-doped titanate nanobelts, while annealing them in air resulted in paramagnetic ordering. Interestingly, by vacuum annealing the undoped titanate nanobelts under the same conditions, superparamagnetic ordering was observed in the resulting anatase $\mathrm{TiO}_{2}$ nanobelts. The electron paramagnetic resonance of this latter sample shows a strong symmetrical signal at $g=2.003$ suggesting some sort of exchange interactions among the localized electrons' spin moments from single electron trapped in oxygen vacancies. (C) 2008 American Institute of Physics. [DOI: 10.1063/1.2944141]
\end{abstract}

The observations of room temperature ferromagnetism in transition metal doped as well as in undoped nanostructural metal oxides have been of great research interest for the past decade. Among the most interesting is the observation of ferromagnetism in several undoped nanoparticle metal oxides reported by Sundaresan et al. ${ }^{1}$ whereby at the nanoscale level, there exists some sort of exchange interactions among the spin moments of the electrons residing on surface oxygen vacant sites of these nanomaterials in mediating long-range magnetic ordering. Titanium oxide nanomaterials doped with small amount of transition metal cobalt have also displayed room temperature ferromagnetism in which Co-doped titanium oxide nanotubes have been the most studied. ${ }^{2-5} \mathrm{Al}-$ though there exist several models to explain the room temperature ferromagnetic phenomenon in diluted magnetic semiconductors, the copresence of cobalt ions and oxygen vacancies and the exchange interactions that arise between them are the more accepted views. In this communication, we report on the magnetic behavior of undoped and Codoped titanium dioxide nanobelts (or nanowires) in their reduced and oxidized states. While the expected room temperature ferromagnetic ordering was observed in the Codoped $\mathrm{TiO}_{2}$ nanobelts, herein we also report on the observation of superparamagnetic ordering in the undoped vacuum reduced $\mathrm{TiO}_{2}$ nanobelts. In a superparamagnetic system, there exist single-domain type magnetic moments which are partially aligned in the presence of an applied field analogous to paramagnetism, but with a much higher susceptibility. Moreover, above a critical temperature $\left(T_{B}\right)$ the remanence and coercivity of the magnetization curve go to zero as the magnetic moments are subjected to thermal relaxation. ${ }^{6}$ We suggest that the unusual magnetic behavior in the reduced sample might be due to the formation of singly electron occupied oxygen vacancies, in which there exist some sort of long-range interactions among the occupying electron spin moments in inducing the superparamagnetic behavior.

The undoped and Co-doped titania nanobelts were synthesized following previously published procedure. $^{7}$ The

${ }^{a)}$ Electronic mail: s_chong@ims.tsukuba.ac.jp. former was prepared from $99.99 \%$ pure commercial $\mathrm{TiO}_{2}$ powder, while the latter nanobelts were prepared by hydrothermally reacting finely crushed powder of a piece of ferromagnetic rutile $\mathrm{Co}_{0.01} \mathrm{Ti}_{0.99} \mathrm{O}_{2}$ (nominal cobalt concentration) single crystal ${ }^{8}$ in $10 \mathrm{~mol} / 1 \mathrm{NaOH}(\mathrm{aq})$ just below $200^{\circ} \mathrm{C}$ for $48 \mathrm{~h}$. Both as-prepared titanate nanobelts were dried overnight in an air oven at $70{ }^{\circ} \mathrm{C}$ before being heat treated under different conditions. Previously, it has been shown that by annealing the titanate nanobelts to $727^{\circ} \mathrm{C}(1000 \mathrm{~K})$ for 3-12 h, pure anatase $\mathrm{TiO}_{2}$ with good crystallinity could be obtained. ${ }^{7}$ Physical and structural phases of the nanobelts were characterized via transmission electron microscope (Jeol 2011) and powder X-ray diffraction (Philips X'pert series). The electron paramagnetic resonance (EPR) spectra were acquired with a Bruker EMX EPR spectrometer at room temperature. The oxidation states of titanium in the nanobelts were determined via x-ray photoelectron spectroscopy (XPS) using $\mathrm{Al} K \alpha$ anode (Perkin-Elmer PHI). Data collection was conducted with a pass energy of $50 \mathrm{eV}$ at $0.1 \mathrm{eV}$ step and $350 \mathrm{~ms} / \mathrm{step}$, while the magnetic properties were measured using a superconducting quantum interference device (SQUID) magnetometer (Quantum Design MPMS).

SQUID magnetometer field dependent magnetization $(M-H)$ measurements at $300 \mathrm{~K}$ [Fig. 1(a)] show the air annealed Co-doped ( 2.5 at. $\%$ by EDX) $\mathrm{TiO}_{2}$ nanobelts have paramagnetic behavior in which the moments are partially aligned in the presence of an applied magnetic field resulting in a net magnetic moment. However, when the Co-doped anatase $\mathrm{TiO}_{2}$ nanobelts were prepared via annealing under vacuum $\left(<1 \times 10^{-2}\right.$ Torr $)$ the $M-H$ curve became irreversible, with a coercive force of $53 \mathrm{Oe}$, indicating ferromagnetism at room temperature. It is well known that annealing metal oxides under reduced atmosphere will create oxygen vacancies in the lattice sites, particularly on the surface but also in the bulk. In the case of $\mathrm{TiO}_{2}$, the presence of these oxygen vacancies is pivotal to its various functional properties. ${ }^{9}$ EPR has been an invaluable tool in identifying electrons and holes (herein denoted as active species) that are trapped in these defect sites, enabling the mapping of the different types of oxygen vacancies that exist on both the surface and bulk of $\mathrm{TiO}_{2}{ }^{10,11}$ The EPR spectra in Fig. 1(b) 


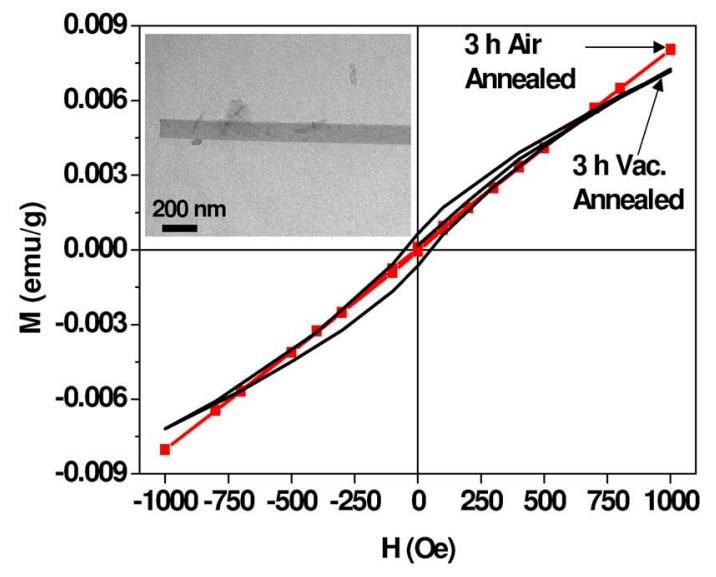

(a)

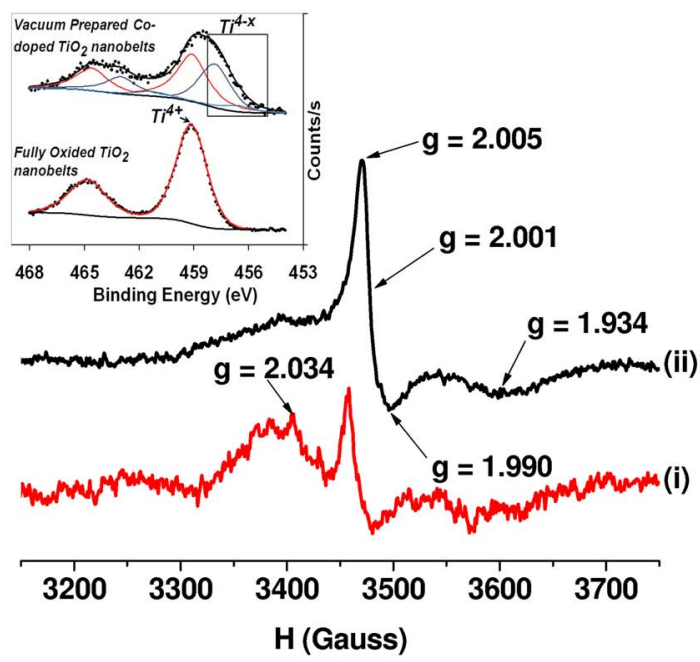

(b)

FIG. 1. (Color online) $M-H$ measurements (a) at $300 \mathrm{~K}$ of Co-doped anatase $\mathrm{TiO}_{2}$ annealed under air (i) and vacuum (ii), and the corresponding EPR signals (b). The inset in (a) shows a typical transmission electron microscopy image of a Co-doped $\mathrm{TiO}_{2}$ nanobelt, while that in (b) shows the comparison between the XPS Ti $2 p$ of a fully oxidized $\mathrm{TiO}_{2}$ nanobelts and the vacuum prepared Co-doped $\mathrm{TiO}_{2}$ nanobelts.

show that both the air and vacuum annealed Co-doped nanobelts contained stable active species residing on bulk and surface defect sites as well as on oxygenated sites primarily on the surface. The EPR signals with $g$ value of $\sim 2.001-2.005$ are due to single-electron trapped in oxygen vacancies, ${ }^{12,13}$ while surface plus bulk $\mathrm{Ti}^{3+}$ species have $g$ values of 1.99-1.93. ${ }^{10,11}$ The latter reduced titanium species was also observed by $\mathrm{Ti} 2 p$ core-level x-ray photoelectron emission spectrum [inset of Fig. 1(b)]. Compared to the fully oxidized undoped $\mathrm{TiO}_{2}$ nanobelts, a shoulder is clearly observable on the lower binding energies side of the XPS $\mathrm{Ti}^{4+}$ peak at $459.3 \mathrm{eV}$, which can be attributed to the presence of $\mathrm{Ti}^{4-x}$ species. ${ }^{14}$ The broad EPR signals on the left side of the sharp peak are most likely due to those of surface oxygenated active species. ${ }^{11,15}$ This assignment is consistent with our experimental observations where the intensities of these signals are always higher in all air annealed samples, but relatively lower in all samples that were prepared under vacuum annealing.

Nanostructural titanium oxides are well known for their high defect content that are able to trap electrons which conDownloaded 09 Jul 2008 to 130.158 .56 .123 . Redistribution subject
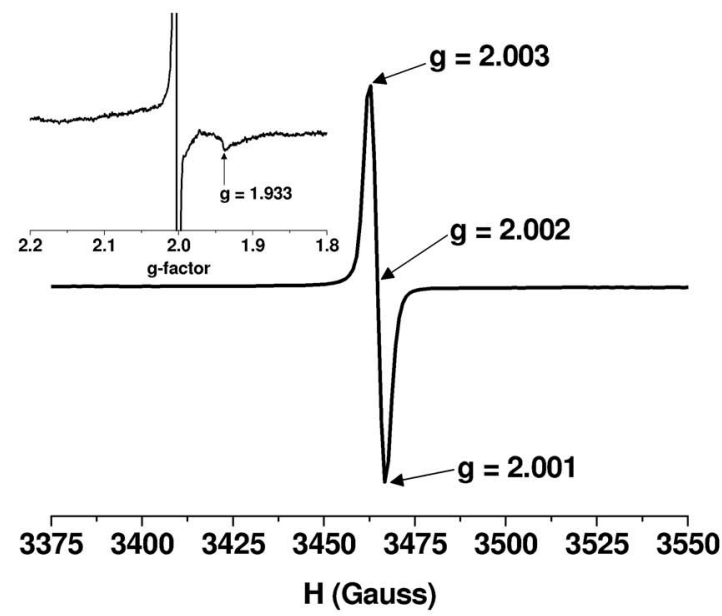

(a)

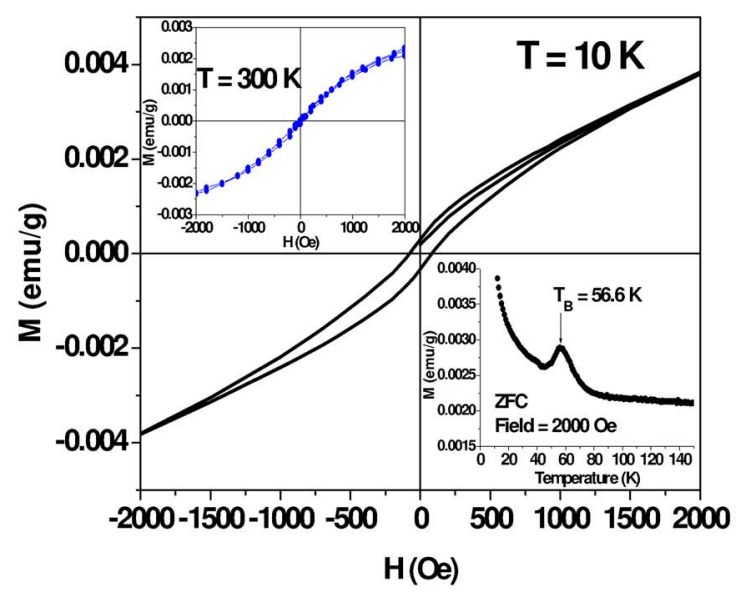

(b)

FIG. 2. (Color online) EPR spectrum acquired at $300 \mathrm{~K}$ (a), and the corresponding field dependent magnetization curves at 300 and $10 \mathrm{~K}$ (b) for the undoped $\mathrm{TiO}_{2}$ nanobelts prepared via vacuum annealing. The other inset in (b) shows the ZFC measurement taken at 2000 Oe.

tributes to their enhanced functional behaviors. ${ }^{16,17}$ As previously mentioned, the report on room temperature ferromagnetism on nanoparticles metal oxides attributed to the exchange interactions among localized electron spin moments originated from electrons trapped on surface oxygen vacancies is of great interest, since unlike diluted magnetic semiconductors these nanoparticles contained no magnetic impurities. ${ }^{1}$ With this in mind, we investigated the magnetic behaviors of undoped $\mathrm{TiO}_{2}$ anatase nanobelts prepared from vacuum annealing of titanate nanobelts. EPR [Fig. 2(a)] of these reduced $\mathrm{TiO}_{2}$ nanobelts shows a single sharp symmetrical signal $(g=2.003)$ which can be attributed to singleelectron trapped on oxygen vacant sites, also known as singly occupied $F^{+}$-centers. ${ }^{12,13}$ Studies carried out on Codoped titanate nanotubes have shown the importance of this type of oxygen vacancies in mediating room temperature ferromagnetism, ${ }^{2-5}$ with the widely accepted view of $F$-centers induced ferromagnetic coupling between $\mathrm{Co}^{2+}$ via a donor impurity band exchange model. ${ }^{18,19}$ The enlarged plot of the same graph (inset) also reveals the presence of active species trapped on $\mathrm{Ti}^{3+}$ sites $(g=1.933)$ but the amount of these species is significantly smaller compared to that of the former.

to AIP license or copyright; see http://apl.aip.org/apl/copyright.jsp 
The magnetization curve of the reduced $\mathrm{TiO}_{2}$ nanobelts recorded at room temperature [inset of Fig. 2(b)] shows that these nanobelts exhibit superparamagnetic behavior. This was further confirmed via zero-field-cooled (ZFC) measurement where a transition from the thermally agitated "random" state to a state where the magnetic moments are blocked below the "blocking temperature" $\left(T_{B}\right)$ at $56.6 \mathrm{~K}$ was observed. A hysteresis loop was clearly observed when the $M-H$ curve was measured below the blocking temperature, as shown in Fig. 2(b). Owing to the presence of a large amount of singly occupied $\mathrm{F}^{+}$-centers, and with the absence of any impurities, it is therefore very likely that the observed superparamagnetic ordering is due to some sort of exchange interactions among the unpaired electron spin moments of these $F^{+}$-centers. Since the shape of these $\mathrm{TiO}_{2}$ nanobelts is highly anisotropic with preferential exposed faces, it is therefore worth further investigation into the size and shape dependence of $\mathrm{TiO}_{2}$ nanostructural materials in order to elucidate the influence that these factors may have in governing the interactions among the electron spin moments, and therefore the resulting magnetic behavior.

In summary, we have shown the importance of the presence of certain type of oxygen vacancies in governing the magnetic behaviors of Co-doped and undoped $\mathrm{TiO}_{2}$ nanobelts. In the former case, the presence of these vacancies together with cobalt doping mediated ferromagnetic ordering at room temperature. In the case of vacuum reduced undoped anatase $\mathrm{TiO}_{2}$ nanobelts, it is proposed that the exchange interactions among the spin moments of the unpaired electrons occupying in this type of oxygen vacancies, in forming $F^{+}$-centers, mediated superparamagnetic ordering in the sample.

This work is supported by the Japan Society for the Promotion of Science (JSPS) postdoctoral fellowship. The authors would also like to thank K. Yamaki for providing the Co-doped rutile single crystal in this study.

${ }^{1}$ A. Sundaresan, R. Bhargavi, N. Rangarajan, U. Siddesh, and C. N. R. Rao, Phys. Rev. B 74, 161306 (2006).

${ }^{2}$ D. Wu, Y. Chen, J. Liu, X. Zhao, A. Li, and N. Ming, Appl. Phys. Lett. 87, 112501 (2005).

${ }^{3}$ C. Huang, X. Liu, Y. Liu, and Y. Wang, Chem. Phys. Lett. 432, 468 (2006).

${ }^{4}$ C. Huang, X. Liu, L. Kong, W. Lan, Q. Su, and Y. Wang, Appl. Phys. A: Mater. Sci. Process. 87, 781 (2007).

${ }^{5}$ X. W. Wang, X. P. Gao, G. R. Li, L. Gao, and T. Y. Yan, Appl. Phys. Lett. 91, 143102 (2007).

${ }^{6}$ J. C. Mallinson in Magnetic Properties of Materials, edited by J. Smit (McGraw-Hill Book Company, New York, 1971), Chap. 7.

${ }^{7}$ S. V. Chong, N. Suresh, J. Xia, N. Al-Salim, and H. Idriss, J. Phys. Chem. C 111, 10389 (2007).

${ }^{8}$ K. Yamaki, N. Shimizu, E. Kita, T. Mochiku, H. Fujii, K. Yamada, S. Itoh, and K. Kadowaki, Phys. Status Solidi C 3, 4127 (2006).

${ }^{9}$ T. L. Thompson and John T. Yates, Jr., Chem. Rev. (Washington, D.C.) 106, 4428 (2006).

${ }^{10}$ J. M. Cho, W. J. Yun, J.-K. Lee, H. S. Lee, W. W. So, S. J. Moon, Y. Jia, H. Kulkarni, and Y. Wu, Appl. Phys. A: Mater. Sci. Process. 88, 751 (2007).

${ }^{11}$ Y. Nakaoka and Y. Nosaka, J. Photochem. Photobiol., A 110, 299 (1997).

${ }^{12}$ S. Zhang, W. Li, Z. Jin, J. Yang, J. Zhang, Z. Du, and Z. Zhang, J. Solid State Chem. 177, 1365 (2004).

${ }^{13}$ M. Sterrer, E. Fischbach, T. Risse, and H.-J. Freund, Phys. Rev. Lett. 94, 186101 (2005).

${ }^{14}$ L. Q. Wang, D. R. Baer, M. H. Engelhard, and A. N. Shultz, Surf. Sci. 344, 237 (1995).

${ }^{15}$ J. M. Coronado, A. J. Maira, J. C. Conesa, K. L. Yeung, V. Augugliaro, and J. Soria, Langmuir 17, 5368 (2001).

${ }^{16}$ G. Wang, Q. Wang, W. Lu, and J. Li, J. Phys. Chem. B 110, 22029 (2006).

${ }^{17}$ K. Pan, Q. Zhang, Q. Wang, Z. Liu, D. Wang, J. Li, and Y. Bai, Thin Solid Films 515, 4085 (2007).

${ }^{18}$ J. M. D. Coey, M. Venkatesan, and C. B. Fitzgerald, Nat. Mater. 4, 173 (2005).

${ }^{19}$ D. Pan, G. Xu, L. Lv, Y. Yong, X. Wang, J. Wan, G. Wang, and Y. Sui, Appl. Phys. Lett. 89, 082510 (2006). 